\title{
High-precision mass measurements of nickel, copper, and gallium isotopes and the purported shell closure at $N=\mathbf{4 0}$
}

\author{
C. Guénaut, ${ }^{1, *}$ G. Audi, ${ }^{1}$ D. Beck,${ }^{2}$ K. Blaum,${ }^{2,3}$ G. Bollen, ${ }^{4}$ P. Delahaye,${ }^{5}$ F. Herfurth,${ }^{2}$ A. Kellerbauer,${ }^{5, \dagger}$ \\ H.-J. Kluge, ${ }^{2,6}$ J. Libert, ${ }^{7}$ D. Lunney, ${ }^{1}$ S. Schwarz, ${ }^{4}$ L. Schweikhard,${ }^{8}$ and C. Yazidjian ${ }^{2}$ \\ ${ }^{1}$ CSNSM-IN2P3-CNRS, F-91405 Orsay-Campus, France \\ ${ }^{2}$ GSI, Planckstraße 1, D-64291 Darmstadt, Germany \\ ${ }^{3}$ Johannes Gutenberg-Universität, Institut für Physik, D-55099 Mainz, Germany \\ ${ }^{4}$ NSCL, Michigan State University, East Lansing, Michigan 48824, USA \\ ${ }^{5}$ CERN, Physics Department, $\mathrm{CH}-1211$ Genève 23, Switzerland \\ ${ }^{6}$ Physikalisches Institut, Universität Heidelberg, D-69120 Heidelberg, Germany \\ ${ }^{7}$ Institut de Physique Nucléaire, IN2P3-CNRS, F-91406 Orsay-Campus, France \\ ${ }^{8}$ Institut für Physik, Ernst-Moritz-Arndt-Universität, D-17487 Greifswald, Germany
}

(Received 14 December 2006; published 9 April 2007)

\begin{abstract}
High-precision mass measurements of more than 30 neutron-rich nuclides around the $Z=28$ closed proton shell were performed with the triple-trap mass spectrometer ISOLTRAP at ISOLDE/CERN to address the question of a possible neutron shell closure at $N=40$. The results for ${ }^{57,60,64-69} \mathrm{Ni}(Z=28),{ }^{65-74,76} \mathrm{Cu}(Z=29)$, and ${ }^{63-65,68-78} \mathrm{Ga}(Z=31)$ have a relative uncertainty of the order of $10^{-8}$. In particular, the mass of ${ }^{76} \mathrm{Cu}$ was measured for the first time. We analyze the resulting mass surface for signs of magicity, comparing the behavior of $N=40$ with that of known magic numbers and with midshell behavior. While the classic indications from the mass surface show no evidence for a shell closure at $N=40$, there is evidence for a weak-and very localized-effect for $Z=28$, consistent with findings from nuclear spectroscopy studies.
\end{abstract}

DOI: 10.1103/PhysRevC.75.044303 PACS number(s): 21.10.Dr, 21.60.Cs, 27.50.+e, 32.10.Bi

\section{INTRODUCTION}

A property crucial to the understanding of the nuclear system is the behavior of its shell structure as a function of the varying composition of protons and neutrons. The fact that shell structure seems to be modified in systems where the number of neutrons $N$ and the number of protons $Z$ are unbalanced (i.e., far from the equilibrium region of stable nuclides) is one of the key questions facing today's nuclear physics research.

Magic numbers have been found to vanish in certain regions of the chart of nuclides, the first one being $N=20$ for sodium [1] and later, magnesium [2]. More recently, $N=8$ [3,4] and $N=28[5,6]$ have also disappeared. Conversely, "new" magic numbers such as $N=16$ [3] and $N=32$ [7-9] have been found. One case of particular interest is that of $N=40$ because of the unexpected events that have transpired since the first studies in 1982. At that time, Bernas et al. [10] showed that the first excited state of ${ }_{28}^{68} \mathrm{Ni}_{40}$ was $0^{+}$, establishing a new case of $2^{+}$and $0^{+}$inversion. This was compared with the case of ${ }_{20}^{40} \mathrm{Ca}_{20}$, a doubly magic nuclide [11] where such an inversion was known. Consequently, Bernas et al. concluded ${ }^{68} \mathrm{Ni}$ to be doubly magic.

In 1995, Broda et al. [12] published a comprehensive summary of spectroscopy work performed since 1982 and

${ }^{*}$ Corresponding author; present address: NSCL, Michigan State University, East Lansing, MI 48824, USA; E-mail address: guenaut@nscl.msu.edu

${ }^{\dagger}$ Present address: Max Planck Institut für Kernphysik, Postfach 103980, D-69029 Heidelberg, Germany. elaborated the excited spectrum of ${ }^{68} \mathrm{Ni}$, finding the first excited state to be $0^{+}$(as in Bernas et al. [10]), $2^{+}$as the second excited state, and a $5^{-}$isomeric state. They concluded that since ${ }^{68} \mathrm{Ni}$ showed the same structure as the spherical ${ }^{90} \mathrm{Zr}$ nuclide, ${ }^{68} \mathrm{Ni}$ was also spherical, implying a significant subshell closure at $N=40$. Shell-model predictions of isomeric states near magic nuclides motivated the experimental investigations of Grzywacz et al. [13] in 1998. They discovered many isomeric states in the vicinity of ${ }^{68} \mathrm{Ni}$, further strengthening the case for its doubly magic character. In $1999, \beta$-decay studies were carried out by Hannawald et al. [14], who found long half-lives for the neighboring isotones (copper, manganese) at $N=40$, indicating an increase in collectivity. However, $\beta$-decay studies by Mueller $e t$ al. [15] the same year showed that the stabilizing effect of $N=40$ disappeared when moving away from ${ }^{68} \mathrm{Ni}$.

The powerful tool of Coulomb excitation was brought to bear on ${ }^{68} \mathrm{Ni}$ in 2002 when Sorlin et al. [16] measured the $B(E 2)$ value (which is the probability of transition between the ground state $0^{+}$and the excited state $\left.2^{+}\right) . B(E 2)$ is expected to be small for magic nuclides, which are difficult to excite, and to be large for deformed nuclides. The measured $B(E 2)$ value was unexpectedly small, reinforcing the magic nature of ${ }^{68} \mathrm{Ni}$. Sorlin et al. explained that the reason the magic nature of $N=40$ was not visible from the mass surface was that the $N=40$ shell closure was quenched. However, a concerted theoretical effort published by Langanke et al. [17] argued agaist the doubly magic nature of ${ }^{68} \mathrm{Ni}$, noting that the "missing" $B(E 2)$ strength lies at much higher energy ( $>4 \mathrm{MeV}$ ).

According to Bohr and Mottelson [18], "In terms of the expansion of the total binding energy, the shell structure appears as a small correction compared to the surface energy.... 
Despite the smallness of these effects on the scale of the total nuclear energy, they are of decisive importance for the structure of the low-energy nuclear spectra." In light of these conflicting experimental and theoretical signatures as well as the relatively large uncertainty on the binding energies in this interesting region, high-precision mass measurements were carried out with the mass spectrometer ISOLTRAP in an attempt to clarify this situation. The first direct mass measurements in this region were performed on the time-of-flight isochronous (TOFI) spectrometer at Los Alamos, reported in 1994 [19] and 1998 [20]. Though they discussed the issue of the magicity at $N=40$, the uncertainty of the results was insufficient to draw any conclusions. The most accurate mass measurements today are performed in Penning traps [21,22], and ISOLTRAP at CERN has pioneered the application to radioactive nuclides $[23,24]$. The experimental setup of ISOLTRAP is presented in Sec. II, and the measurements in the region of $N=40$ and their evaluation are described in Sec. III. A comparison to mass models follows in Sec. IV, and the question of $N=40$ is discussed in light of the new results in the last section.

\section{ISOLTRAP SETUP}

\section{A. Experimental setup}

ISOLTRAP is a high-precision Penning-trap mass spectrometer, located at CERN's ISOLDE facility [25] which delivers mass-separated beams of radionuclides. ISOLTRAP is composed of three main parts (see Fig. 1). First, a linear gas-filled radio-frequency quadrupole (RFQ) trap, used as cooler and buncher, adapts the $60-\mathrm{keV}$ ISOLDE ion beam to the ISOLTRAP requirements with respect to kinetic energy, time structure, and beam emittance [26]. The second part is a gas-filled, cylindrical Penning trap [27] in which a mass-selective helium buffer-gas cooling technique [28] with a resolving power of up to $10^{5}$ is used for isobaric cleaning. This preparation trap is installed in a $B=4.7 \mathrm{~T}$ superconducting magnet. Finally, the cooled ion bunch is transferred to the precision Penning trap for isomeric separation (when required) and mass measurement. The precision Penning trap is installed in a second superconducting magnet ( $B=5.9 \mathrm{~T})$. The mass is determined by measuring the true cyclotron frequency $v_{c}=\mathrm{qB} /(2 \pi m)$ of the stored ion (see next paragraph). The magnetic field $B$ is determined from a measurement of the cyclotron frequency of a reference ion whose mass is well known. The setup also includes an off-line ion source to produce stable ions, used as reference masses.

\section{B. Mass measurement procedure}

Ion confinement in a Penning trap is based on the application of an electrostatic field and a magnetic field to store ions in the axial and radial directions, respectively. The ion motion in a Penning trap is a superposition of three independent harmonic oscillator modes, one in the axial direction with frequency $v_{z}$ and two in the radial direction, i.e., the cyclotron motion with reduced frequency $v_{+}$and the magnetron motion with

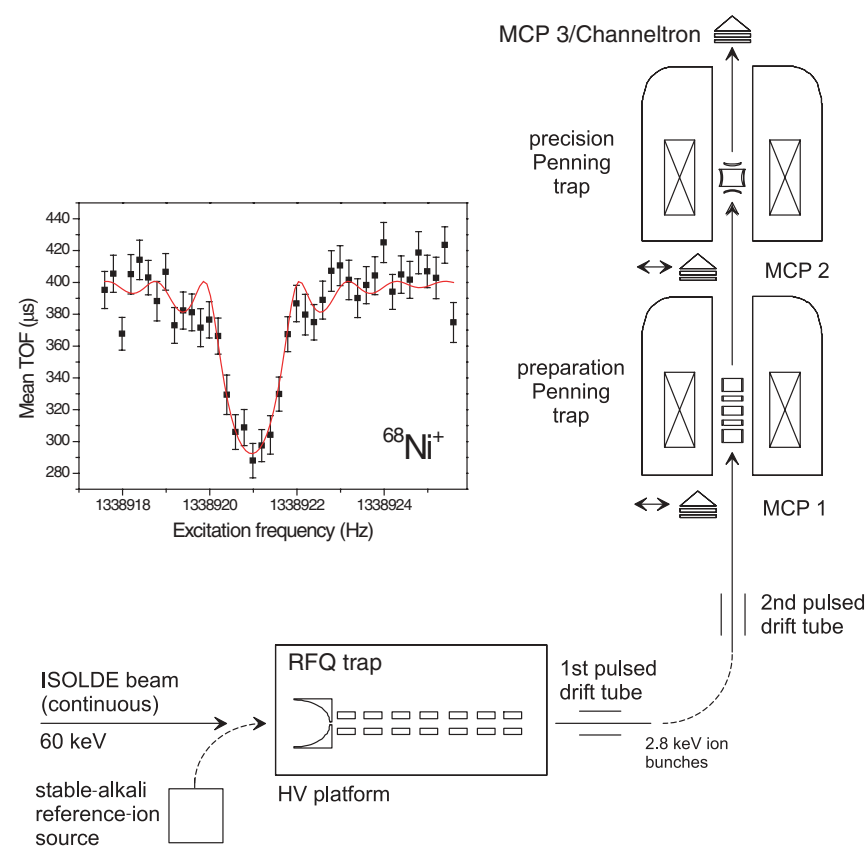

FIG. 1. (Color online) Sketch of experimental setup of ISOLTRAP mass spectrometer, including the main parts: a gas-filled linear radio-frequency quadrupole (RFQ) trap for capturing and preparing the ISOLDE beam, a gas-filled cylindrical Penning trap for isobaric separation, and a hyperbolic Penning trap for the mass measurement. Microchannel plate (MCP) detectors are used to monitor the ion transfer and to measure the extracted-ion time of flight (TOF) together with the channeltron detector. Inset presents a TOF cyclotron resonance for radioactive ${ }^{68} \mathrm{Ni}^{+}$ions.

frequency $v_{-}[29,30]$. In a purely quadrupolar electric field, the frequencies are related as

$$
v_{c}=v_{+}+v_{-} .
$$

Ion beams are alternatively delivered from ISOLDE or from an off-line ion source and injected into the RFQ, mounted on a $60-\mathrm{keV}$ pedestal, where they are cooled and bunched. The ion bunch from the RFQ is sent to the preparation trap. Ion collisions with the buffer gas inside this trap first cool the axial motion. A dipolar excitation with a frequency $\nu_{-}$is then applied to increase the magnetron radius of all ion species, making it larger than the exit hole of the trap. To select the ions of interest, an azimuthal quadrupole radio-frequency electric field at frequency $v_{c}$ is applied which couples the radial modes. Since one mode is cooled by the gas, the radius is reduced and the ion cloud is centered. In this way, the trap works as an isobar separator with a resolving power $R=m / \Delta m$ of $10^{4}$ to $10^{5}$ [27].

The purified ion beam is transferred to the precision trap, where different excitations are performed. A phase-sensitive dipolar excitation at $v_{-}$is applied to increase the magnetron radius of the ion motion [31]. If there are contaminants (isobars or isomers), a second, mass-dependent dipolar excitation is performed at $v_{+}$to remove them [32]. Finally, an azimuthal quadrupole radio-frequency (RF) field is applied to convert the initial magnetron motion into cyclotron motion. At $v_{\mathrm{RF}}=v_{c}$, a full conversion is obtained, leading to an increase of the orbital 
magnetic moment $\mu$ and the associated radial kinetic energy $E=\mu B$ [33]. After ejection at low axial energy, ions pass the inhomogeneous part of the magnetic field on their way to an MCP detector (recently replaced by a channeltron detector [34]) at the top of the setup. Since the axial acceleration in this fringe field is proportional to $\mu \cdot \partial B / \partial z$, the shortest time of flight (TOF) is observed for $v_{\mathrm{RF}}=v_{c}$ [35].

The mass resolution in the precision trap depends strongly on the conversion time used for the excitation. The line width $\Delta v$ of the resonance is mainly determined by the duration of the applied RF field $\left(T_{\mathrm{RF}}\right)$ used to couple the two radial motions. The relation is [33]

$$
\Delta v(\mathrm{FWHM}) \approx \frac{0.9}{T_{\mathrm{RF}}} .
$$

The statistical precision in the cyclotron frequency determination is given by [36]

$$
\frac{\delta v}{v} \propto \frac{1}{v T_{\mathrm{RF}} \sqrt{N}},
$$

with $N$ being the number of ions and $R=v T_{\mathrm{RF}}$ the resolving power. With sufficiently long excitation times (a few seconds), a resolving power of up to $10^{7}$ can be reached. As an example of a cyclotron frequency measurement, the inset of Fig. 1 presents the TOF-resonance curve of one of the two measurements of radioactive ${ }^{68} \mathrm{Ni}$. The mean $\mathrm{TOF}$ of the ions as a function of the applied RF is shown. The solid line is a fit of the wellknown line shape [30] to the data points. This measurement was performed with about 1000 ions, using an excitation time $T_{\mathrm{RF}}=900 \mathrm{~ms}$, resulting in a resolving power of $1.1 \times 10^{6}$ and a relative frequency uncertainty of $\delta v / v=6 \times 10^{-8}$.

\section{MEASUREMENTS OF NI, CU, AND GA ISOTOPES}

The nuclides ${ }^{57,60,64-69} \mathrm{Ni},{ }^{65-74,76} \mathrm{Cu}$, and ${ }^{63-65,68-78} \mathrm{Ga}$ have been investigated with ISOLTRAP. They were produced at ISOLDE by bombarding a uranium carbide (UC) target with 1.4-GeV protons from CERN's proton synchroton booster. The ionization was achieved for gallium with a tungsten (W) surface ionization ion source and for copper and nickel with the resonance ionization laser ion source (RILIS) [37]. ISOLDE's general purpose separator (GPS), with a mass resolving power of about 1000 was used. The proton-rich isotopes ${ }^{63-65} \mathrm{Ga}$ were measured in a different experiment using a $\mathrm{ZrO}$ target and ISOLDE's high-resolution separator (HRS), which has a mass-resolving power of about 3000 . Both targets were bombarded using pulses containing up to $3 \times 10^{13}$ protons.

The yields of nickel and copper were fairly intense at about $10^{5}$ ions/s. The efficiency of ISOLTRAP is better than $1 \%$, so a beam gate was used to limit the number of ions sent to the precision trap and minimize ion-ion interactions that cause frequency shifts. The typical number of ions simultaneously stored in the precision trap was between 1 and 8 .

Despite the good yields of nickel and copper nuclides, up to three orders of magnitude more surface-ionized gallium was present. For the measurement of ${ }^{68} \mathrm{Ni}$ shown in Fig. 1, a cleaning of ${ }^{68} \mathrm{Ga}$ was applied in the preparation trap. The ratio between the yield of ${ }^{68} \mathrm{Ga}$ and ${ }^{68} \mathrm{Ni}$ was "only" a factor of 10 , which was low enough to allow an effective cleaning. This ratio was higher farther from stability and prevented the measurement of more neutron-rich nickel and copper, since the preparation trap was saturated by the gallium isobars. Similarly, a significant contamination of titanium oxide prevented the measurement of more proton-rich gallium isotopes, and the presence of rubidium isobars made the measurement of more neutron-rich gallium isotopes impossible.

The result from the data analysis is the ratio $v_{c \text {,ref }} / v_{c}$ [36], since the atomic mass $m$ of the ions is calculated from the ratio between the cyclotron frequency of the reference ion $v_{c \text {,ref }}$ and the cyclotron frequency of the ion of interest $v_{c}$, the atomic mass of the reference ${ }^{85} \mathrm{Rb}$ [38], and the electron mass $m_{e}$ :

$$
m=\frac{v_{c, \text { ref }}}{v_{c}}\left(m_{85 \mathrm{Rb}}-m_{e}\right)+m_{e}
$$

All the results were evaluated in order to include them in the atomic-mass evaluation (AME) table [39]. The table of atomic masses results from an evaluation of all available experimental data on masses, including direct measurements as well as decay and reaction studies. The AME forms a linked network and uses a least-squares adjustment to derive the atomic masses. Among all nuclear ground-state properties, such an evaluation is unique to mass measurements.

The mass values from the present measurements are presented in Tables I (Ni), II (Cu), and III (Ga). These tables give the ratio of the cyclotron frequency of the ${ }^{85} \mathrm{Rb}^{+}$[38] reference mass to that of the ion of interest. The corresponding uncertainty takes into account a statistical uncertainty depending on the number of ions, and a systematic error [36]. The derived mass excess value is indicated for comparison with the AME tables from 1995 and 2003. Since the latest atomic-mass evaluation (AME2003 [40]) includes the data from this work, the influence of the ISOLTRAP measurements is also provided. Among the 36 nuclides measured here, the influence is $100 \%$ for 22 of them.

The nickel results are presented in Table I and Fig. 2. This figure presents the difference between the mass excess measured by ISOLTRAP and the AME1995 values. Note that even for the stable nickel isotopes, the precision of the mass values is improved. With the exception of ${ }^{69} \mathrm{Ni}$ (see below), the results are in good agreement with the 1995 table but much more precise. The masses of ${ }^{57,60,65} \mathrm{Ni}$ agree with the 1995 table within the error bars and were measured with the same order of uncertainty. The combination of the previous value and the ISOLTRAP measurement reduces the final uncertainty. The results contributing to the ${ }^{69} \mathrm{Ni}$ mass value are presented in Fig. 3. This is a special case because it is in strong disagreement with the AME1995 table [41]: a difference of more than $400 \mathrm{keV}$ was observed. The AME1995 value was derived from a ${ }^{70} \mathrm{Zn}\left({ }^{14} \mathrm{C},{ }^{15} \mathrm{O}\right){ }^{69} \mathrm{Ni}$ reaction [42] and a TOF measurement [19]. The ISOLTRAP value disagrees with the value from the reaction but is in agreement with the TOF measurement. Since the value of ISOLTRAP is much more precise, the AME2003 includes only this value. No indication of the known 3-s isomeric state of ${ }^{69} \mathrm{Ni}$ was seen from our measurements. Koester [43] studied this case in detail and concluded that the isomer would be at least ten times less produced than the ground state. 
TABLE I. ISOLTRAP results for nickel isotopes: nuclide; half-life; frequency ratio $v_{c}$,ref $/ v_{c}$ of nickel isotope to reference nuclide ${ }^{85} \mathrm{Rb}^{+}$[38], corresponding mass excess (ME); mass excess from AME1995; new mass excess from AME2003; influence of the present result on the AME2003 value.

\begin{tabular}{lcccccc}
\hline \hline Isotopes & $\begin{array}{c}\text { Half-life } \\
T_{1 / 2}\end{array}$ & $v_{c, \text { ref }} / v_{c}$ & $\begin{array}{c}\text { ISOLTRAP } \\
\text { ME }(\mathrm{keV})\end{array}$ & $\begin{array}{c}\text { AME1995 } \\
\text { ME (keV) }\end{array}$ & $\begin{array}{r}\text { AME2003 } \\
\text { ME (keV) }\end{array}$ & $\begin{array}{c}\text { Influence on } \\
\text { AME2003 }\end{array}$ \\
\hline${ }^{57} \mathrm{Ni}$ & $35.6 \mathrm{~h}$ & $0.6705736693(316)$ & $-56084.2(2.5)$ & $-56075.5(2.9)$ & $-56082.0(1.8)$ & $52.0 \%$ \\
${ }^{60} \mathrm{Ni}$ & Stable & $0.7057986239(183)$ & $-64472.7(1.4)$ & $-64468.1(1.4)$ & $-64472.1(0.6)$ & $16.6 \%$ \\
${ }^{64} \mathrm{Ni}$ & Stable & $0.7528734602(163)$ & $-67096.9(1.3)$ & $-67095.9(1.4)$ & $-67099.3(0.6)$ & $21.9 \%$ \\
${ }^{65} \mathrm{Ni}$ & $2.5 \mathrm{~h}$ & $0.7646753441(285)$ & $-65129.0(2.3)$ & $-65122.6(1.5)$ & $-65126.1(0.6)$ & $7.8 \%$ \\
${ }^{66} \mathrm{Ni}$ & $55 \mathrm{~h}$ & $0.7764412560(181)$ & $-66006.3(1.4)$ & $-66028.7(16.0)$ & $-66006.3(1.4)$ & $100 \%$ \\
${ }^{67} \mathrm{Ni}$ & $21 \mathrm{~s}$ & $0.7882468785(362)$ & $-63742.7(2.9)$ & $-63742.5(19.1)$ & $-63742.7(2.9)$ & $100 \%$ \\
${ }^{68} \mathrm{Ni}$ & $29 \mathrm{~s}$ & $0.8000274080(377)$ & $-63463.8(3.0)$ & $-63486.0(16.5)$ & $-63463.8(3.0)$ & $100 \%$ \\
${ }^{69} \mathrm{Ni}$ & $12 \mathrm{~s}$ & $0.8118484759(466)$ & $-59978.6(3.7)$ & $-60380(140)$ & $-59979(4)$ & $100 \%$ \\
\hline \hline
\end{tabular}

The copper results are listed in Table II; a comparison with the AME1995 values is given in Fig. 4. An improvement of the mass uncertainty was achieved for all investigated copper isotopes. The values are in good agreement with previous values, except for ${ }^{70} \mathrm{Cu}^{n}$. This important difference is due to an incorrect state assignment. ISOLTRAP's high resolving power of more than $10^{6}$, in combination with $\beta$-decay studies and selective laser ionization, allowed us to perform a clear identification of each state [44]. Moreover, this high resolving power allowed us to resolve isomeric states in ${ }^{68} \mathrm{Cu}$ [45] and to measure them independently. The mass of ${ }^{76} \mathrm{Cu}$ was previously unknown. It is compared with model predictions in Sec. IV.

The gallium results are presented in Table III and in Fig. 5. The ${ }^{68} \mathrm{Ga}$ mass uncertainty, $\delta m / m \approx 5.4 \times 10^{-7}$ is much higher than for all the other nuclides. This is due to the use of a shorter excitation time (100 ms vs $900 \mathrm{~ms}$ for the other nuclides) and to a lack of statistics: only 530 ions were observed, compared with at least 3000 for most of the others. The ISOLTRAP value is still in agreement with the AME1995 value but has no influence. For all other gallium isotopes measured by ISOLTRAP, the uncertainty was decreased. For five of them, it was decreased by more than a factor of 20 , and for ${ }^{63} \mathrm{Ga}$, almost 100 times.

The case of ${ }^{74} \mathrm{Ga}$ was complicated by the possible presence of a 9.5-s isomeric state having an excitation energy of only $60 \mathrm{keV}$ (this accounts for the large AME1995 error bar in Fig. 5). Spectroscopy studies performed in parallel with the mass measurements revealed no indication that the isomer was produced. A 2-s excitation time was used in order to resolve this state in the precision trap, but it was not seen. Moreover, the $z$-class analysis [36] was performed to examine any dependence of the result as a function of ion number, but it revealed no indication of a contaminant. Therefore we are confident that the present result is that of the ground-state mass.

\section{MASS-MODEL PREDICTIONS COMPARED WITH NEW DATA}

Various models and formulas have been developed over the years to predict properties of nuclides, particularly their mass. A review can be found in Ref. [46] where a subset of

TABLE II. Same as Table I, but for copper isotopes. Previously unknown values derived from systematic trends are marked with \#.

\begin{tabular}{lcccccc}
\hline \hline Isotopes $^{\mathrm{a}}$ & $\begin{array}{c}\text { Half-life } \\
T_{1 / 2}\end{array}$ & $v_{c, \text { ref }} / v_{c}$ & $\begin{array}{c}\text { ISOLTRAP } \\
\text { ME (keV) }\end{array}$ & $\begin{array}{c}\text { AME1995 } \\
\text { ME (keV) }\end{array}$ & $\begin{array}{c}\text { AME2003 } \\
\text { ME (keV) }\end{array}$ & $\begin{array}{c}\text { Influence on } \\
\text { AME2003 }\end{array}$ \\
\hline${ }^{65} \mathrm{Cu}$ & Stable & $0.7646483448(139)$ & $-67264.5(1.1)$ & $-67259.7(1.7)$ & $-67263.7(0.7)$ & $36.8 \%$ \\
${ }^{66} \mathrm{Cu}$ & $5.1 \mathrm{~m}$ & $0.7764380632(257)$ & $-66258.8(2.0)$ & $-66254.3(1.7)$ & $-66258.3(0.7)$ & $11.1 \%$ \\
${ }^{67} \mathrm{Cu}$ & $62 \mathrm{~h}$ & $0.7882016658(155)$ & $-67318.8(1.2)$ & $-67300.2(8.1)$ & $-67318.8(1.2)$ & $100 \%$ \\
${ }^{68} \mathrm{Cu}^{g}$ & $31.1 \mathrm{~s}$ & $0.8000008176(199)$ & $-65567.0(1.6)$ & $-65541.9(45.6)$ & $-65567.0(1.6)$ & $100 \%$ \\
${ }^{68} \mathrm{Cu}^{m}$ & $3.7 \mathrm{~m}$ & $0.8000098791(188)$ & $-64850.3(1.5)$ & $-64818(50)$ & $-64845.4(1.7)$ & $50 \%$ \\
${ }^{69} \mathrm{Cu}^{70} \mathrm{Cu}^{g}$ & $2.8 \mathrm{~m}$ & $0.8117756816(174)$ & $-65736.2(1.4)$ & $-65739.9(8.1)$ & $-65736.2(1.4)$ & $100 \%$ \\
${ }^{70} \mathrm{Cu}^{m}$ & $45 \mathrm{~s}$ & $0.8235875816(199)$ & $-62976.1(1.6)$ & $-62960.3(14.5)$ & $-62976.1(1.6)$ & $100 \%$ \\
${ }^{70} \mathrm{Cu}^{n}$ & $33 \mathrm{~s}$ & $0.8235888547(258)$ & $-62875.4(2.0)$ & $-62859(15)$ & $-62875.4(2.0)$ & $100 \%$ \\
${ }^{71} \mathrm{Cu}$ & $6.6 \mathrm{~s}$ & $0.8235906419(272)$ & $-62734.1(2.1)$ & $-62617(15)$ & $-62734.1(2.1)$ & $100 \%$ \\
${ }^{72} \mathrm{Cu}$ & $19 \mathrm{~s}$ & $0.8353679363(194)$ & $-62711.1(1.5)$ & $-62764.2(35.2)$ & $-62711.1(1.5)$ & $100 \%$ \\
${ }^{73} \mathrm{Cu}$ & $6.6 \mathrm{~s}$ & $0.8471819597(182)$ & $-59783.0(1.4)$ & $-60060 \#(200 \#)$ & $-59783.0(1.4)$ & $100 \%$ \\
${ }^{74} \mathrm{Cu}$ & $4.2 \mathrm{~s}$ & $0.8589690332(491)$ & $-58986.6(3.9)$ & $-59160 \#(300 \#)$ & $-58987(4)$ & $100 \%$ \\
${ }^{76} \mathrm{Cu}$ & $1.6 \mathrm{~s}$ & $0.8707837184(779)$ & $-56006.2(6.2)$ & $-55700 \#(400 \#)$ & $-56006(6)$ & $100 \%$ \\
\hline \hline
\end{tabular}

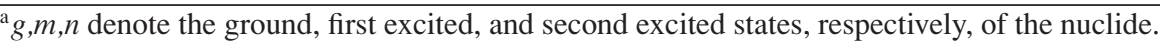


TABLE III. Same as Table I, but for gallium isotopes.

\begin{tabular}{lcccccc}
\hline \hline Isotopes & $\begin{array}{c}\text { Half-life } \\
T_{1 / 2}\end{array}$ & $v_{c, \text { ref }} / v_{c}$ & $\begin{array}{c}\text { ISOLTRAP } \\
\text { ME }(\mathrm{keV})\end{array}$ & $\begin{array}{r}\text { AME1995 } \\
\text { ME (keV) }\end{array}$ & $\begin{array}{r}\text { AME2003 } \\
\text { ME (keV) }\end{array}$ & $\begin{array}{c}\text { Influence on } \\
\text { AME2003 }\end{array}$ \\
\hline${ }^{63} \mathrm{Ga}$ & $32 \mathrm{~s}$ & $0.7412298391(167)$ & $-56547.1(1.3)$ & $-56689.3(100.0)$ & $-56547.1(1.3)$ & $100 \%$ \\
${ }^{64} \mathrm{Ga}$ & $2.6 \mathrm{~m}$ & $0.7529779275(294)$ & $-58834.1(2.3)$ & $-58834.7(3.9)$ & $-58834.3(2.0)$ & $75.2 \%$ \\
${ }^{65} \mathrm{Ga}$ & $15 \mathrm{~m}$ & $0.7647065938(176)$ & $-62657.3(1.4)$ & $-62652.9(1.8)$ & $-62657.2(0.8)$ & $35.6 \%$ \\
${ }^{68} \mathrm{Ga}$ & $68 \mathrm{~m}$ & $0.799981231(431)$ & $-67116.2(34.1)$ & $-67082.9(2.0)$ & $-67086.1(1.5)$ & $0 \%$ \\
${ }^{69} \mathrm{Ga}$ & $\mathrm{Stable}$ & $0.8117302720(193)$ & $-69327.9(1.5)$ & $-69320.9(3.0)$ & $-69327.8(1.2)$ & $65.3 \%$ \\
${ }^{70} \mathrm{Ga}$ & $21 \mathrm{~m}$ & $0.8235125549(272)$ & $-68910.3(2.2)$ & $-68904.7(3.1)$ & $-68910.1(1.2)$ & $31.8 \%$ \\
${ }^{71} \mathrm{Ga}$ & $\mathrm{Stable}$ & $0.8352740255(357)$ & $-70138.9(2.8)$ & $-70136.8(1.8)$ & $-70140.2(1.0)$ & $13.3 \%$ \\
${ }^{72} \mathrm{Ga}$ & $14.1 \mathrm{~h}$ & $0.8470706093(182)$ & $-68590.2(1.4)$ & $-68586.5(2.0)$ & $-68589.4(1.0)$ & $53.0 \%$ \\
${ }^{73} \mathrm{Ga}$ & $4.8 \mathrm{~h}$ & $0.8588335898(208)$ & $-69699.4(1.7)$ & $-69703.8(6.3)$ & $-69699.3(1.7)$ & $100 \%$ \\
${ }^{74} \mathrm{Ga}$ & $8.1 \mathrm{~m}$ & $0.8706314521(469)$ & $-68049.6(3.7)$ & $-68054.0(70.7)$ & $-68050(4)$ & $100 \%$ \\
${ }^{75} \mathrm{Ga}$ & $130 \mathrm{~s}$ & $0.8824032092(305)$ & $-68464.6(2.4)$ & $-68464.2(6.8)$ & $-68464.6(2.4)$ & $100 \%$ \\
${ }^{76} \mathrm{Ga}$ & $33 \mathrm{~s}$ & $0.8942076217(246)$ & $-66296.7(2.0)$ & $-66202.9(90.0)$ & $-66296.6(2.0)$ & $100 \%$ \\
${ }^{77} \mathrm{Ga}$ & $13 \mathrm{~s}$ & $0.9059884728(303)$ & $-65992.4(2.4)$ & $-65874.1(60.0)$ & $-65992.3(2.4)$ & $100 \%$ \\
${ }^{78} \mathrm{Ga}$ & $5.1 \mathrm{~s}$ & $0.9177943761(307)$ & $-63706.6(2.4)$ & $-63662.1(80.1)$ & $-63706.6(2.4)$ & $100 \%$ \\
\hline \hline
\end{tabular}

mass models was singled out for comparison. We have chosen to compare our experimental data with those, as described below.

The venerable Bethe-Weizsäcker mass formula [47,48], was based on the liquid drop model and did not include shell effects. The nuclear mass $m$ is given by

$$
\begin{aligned}
m(N, Z) c^{2}= & Z m_{p} c^{2}+N m_{n} c^{2}-a_{v} A+a_{s} A^{2 / 3} \\
& +a_{c} Z^{2} A^{-1 / 3}+a_{\mathrm{sym}} \frac{(Z-A / 2)^{2}}{A}
\end{aligned}
$$

where $m_{p}$ and $m_{n}$ are the proton and neutron masses, and $A$ the mass number of the nucleus. The parameters are $a_{v}$ the volume term, $a_{s}$ the surface term, $a_{c}$ the Coulomb parameter, and $a_{\mathrm{sym}}$ the asymmetry parameter. Note that the tabulated masses are those of the neutral atoms, not of the bare atomic nuclei. While

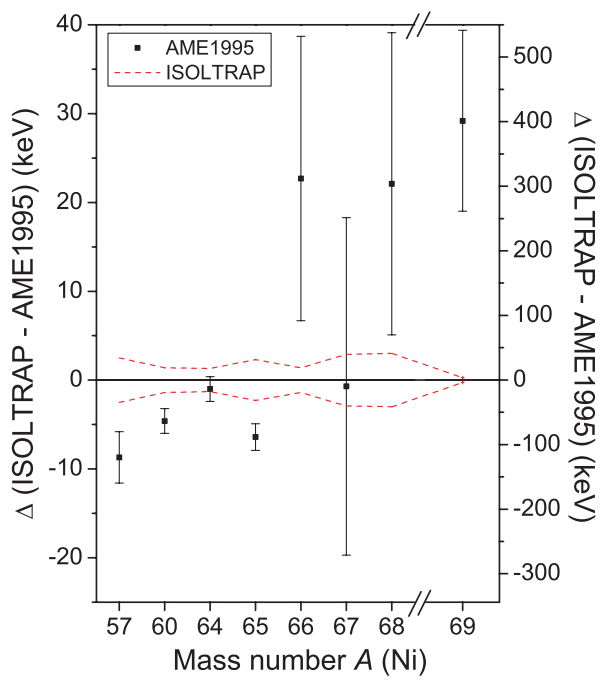

FIG. 2. (Color online) Difference between ISOLTRAP mass excess results for nickel isotopes and the AME1995 values [41]. Dashed lines represent ISOLTRAP error bars. inappropriate for mass predictions, it can play an interesting diagnostic role concerning closed shell effects (see Sec. V D).

For many years, a hybrid approach was adopted for predicting masses based on a combination of the macroscopic liquid drop model and microscopic (e.g., shell) corrections. The most developed form of these so-called mic-mac models is the finite range droplet model (FRDM) [49].

The Duflo-Zuker (DZ) mass formula [50] — a global approach derived from a shell-model Hamiltonian-gives the best fit to the known masses. Shell-model calculations, while well-suited for excitation energies, are less so for mass predictions, although some efforts have been made in this direction [51].

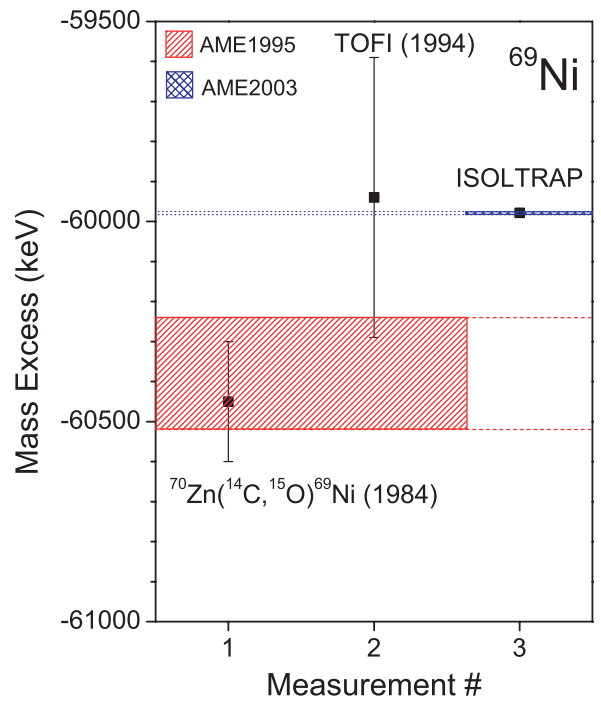

FIG. 3. (Color online) Mass excess of ${ }^{69} \mathrm{Ni}$ determined by the reaction ${ }^{70} \mathrm{Zn}\left({ }^{14} \mathrm{C},{ }^{15} \mathrm{O}\right){ }^{69} \mathrm{Ni}$ [42], and a TOF measurement [19], the resulting AME1995 value [41], and the ISOLTRAP value. The AME2003 value [40] differs by $400 \mathrm{keV}$ with an uncertainty 30 times smaller than the AME1995 value. 


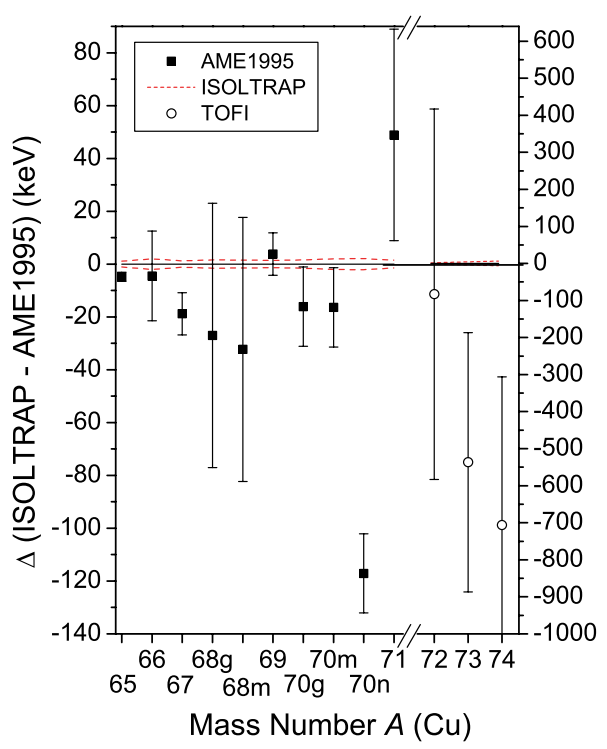

FIG. 4. (Color online) Difference between ISOLTRAP massexcess values for copper isotopes and the 1995 AME values [41]. Dashed lines represent the ISOLTRAP error bars. $g$ denotes ground states and $m, n$ isomeric states. Mass measurements from Bai et al. [20] from 1998 are represented by open circles. ISOLTRAP improves the mass uncertainty of ${ }^{72-74} \mathrm{Cu}$ over 100 times.

In the last few years, Hartree-Fock Bogoliubov (HFB) calculations have been applied to the construction of complete mass tables. Skyrme forces have traditionally aimed at predicting a wide range of nuclear properties [52-55]. The first microscopic Skyrme-force mass formula HFBCS-1 [56,57] was rapidly superseded by HFB-1 [58] which, in turn, was considerably revised, resulting in HFB-2 [59]. A systematic study of the different adjustable parameters followed, resulting in a series of formulas up to HFB-9 [60-63].

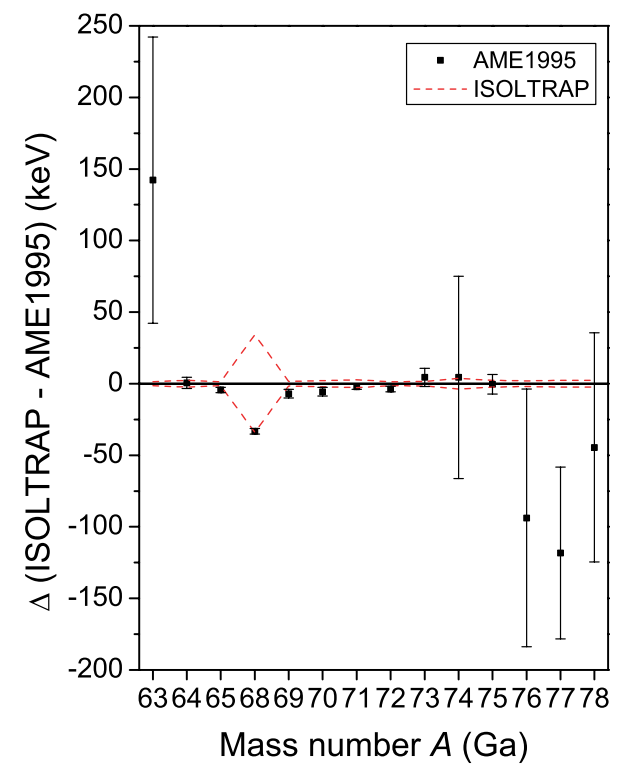

FIG. 5. (Color online) Difference between ISOLTRAP massexcess values for gallium isotopes and the 1995 AME values [41]. Dashed lines represent the ISOLTRAP error bars.
TABLE IV. Root-mean-square deviation $\sigma_{\text {rms }}$ (in $\mathrm{MeV}$ ) for different models: Duflo-Zuker (DZ) mass formula, finite range droplet model (FRDM), and Hartree-Fock Bogoliubov (HFB) calculations, performed with the AME tables of 1995 and 2003 (the latter includes the present ISOLTRAP data). Calculations were made for nickel, copper, and gallium isotopes measured by ISOLTRAP. First two rows present the calculations for all nuclides; following rows describe the results for each isotopic chain separately.

\begin{tabular}{lccccc}
\hline \hline Nuclide & Table & DZ & FRDM & HFB-2 & HFB-8 \\
\hline $\mathrm{Ni}, \mathrm{Cu}, \mathrm{Ga}$ & AME95 & 0.434 & 0.555 & 0.843 & 0.550 \\
$\mathrm{Ni}, \mathrm{Cu}, \mathrm{Ga}$ & AME03 & 0.451 & 0.555 & 0.801 & 0.530 \\
$\mathrm{Ni}$ & AME95 & 0.623 & 0.445 & 1.211 & 0.732 \\
$\mathrm{Ni}$ & AME03 & 0.640 & 0.476 & 1.174 & 0.678 \\
$\mathrm{Cu}$ & AME95 & 0.426 & 0.471 & 0.644 & 0.601 \\
$\mathrm{Cu}$ & AME03 & 0.451 & 0.530 & 0.626 & 0.563 \\
$\mathrm{Ga}$ & AME95 & 0.280 & 0.644 & 0.654 & 0.375 \\
$\mathrm{Ga}$ & AME03 & 0.291 & 0.614 & 0.648 & 0.384 \\
\hline \hline
\end{tabular}

Therefore, in addition to being compared with DZ and FRDM predictions, the ISOLTRAP results are compared with those of HFB-2 and the recent HFB-8 (HFB-9 did not change the mass predictions appreciably).

One characterization of a model is the root-mean-square (rms) deviation from the mass values to which its parameters were fitted, defined by

$$
\sigma_{\mathrm{rms}}=\frac{1}{N} \sqrt{\sum_{i=1}^{N}\left(m_{\mathrm{exp}}^{i}-m_{\mathrm{th}}^{i}\right)^{2}},
$$

where $N$ is the number of experimental $m_{\exp }$ and theoretical $m_{\text {th }}$ masses being compared. A more complete description of the rms deviation, including errors, can be found in Ref. [46]. Table IV shows $\sigma_{\text {rms }}$ for the models compared with the AME95 table [41], which does not include the present ISOLTRAP results, and with AME03 [40], which does. Our results improved the overall agreement for the HFB models, worsened it for the DZ mass formula, and caused no change for FDRM. Examining the isotopic chains individually, we see that in all cases, the HFB models improved and the DZ model worsened. For the FRDM, the better fit for the gallium isotopes counters the worse fit for copper and nickel. The differences are admittedly small (between $1 \%$ and $10 \%$ ). While it is tempting to conclude that the comparison of the $\sigma_{\text {rms }}$ might demonstrate the positive evolution of HFB-2 to HFB-8, it is important to recall that unlike FRDM and DZ, HFB-8 was adjusted to the masses of the AME03.

Of particular interest for mass models is to compare predictions as far as possible from what is already known. In the case of the copper isotopes presented here, one of them $\left({ }^{76} \mathrm{Cu}\right)$ has been measured for the first time. The differences of the new ISOLTRAP copper masses with respect to the above-mentioned models are shown in Fig. 6.

Despite going significantly farther from stability, it is difficult to assess which model does a better job. The one closest to the new mass of ${ }^{76} \mathrm{Cu}$ is HFB-8; however, the other models are not far away. The rms errors on just the 


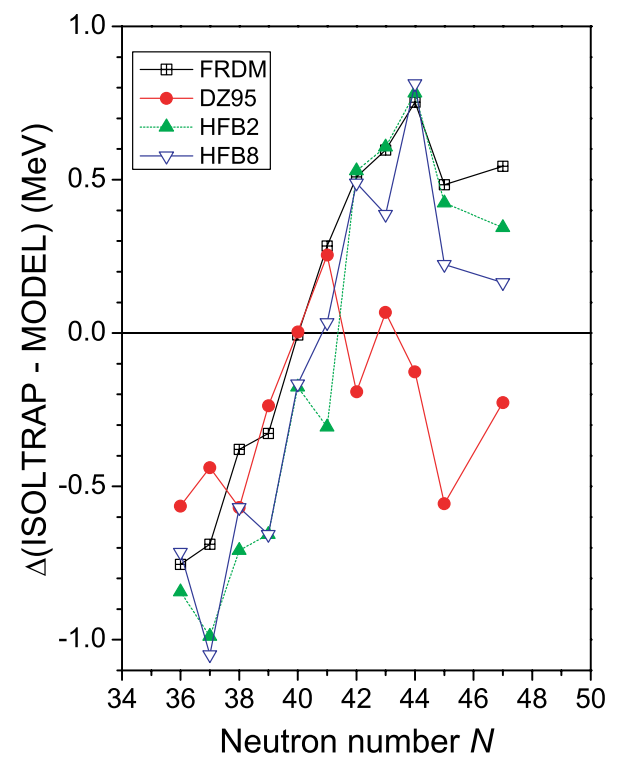

FIG. 6. (Color online) Mass difference between ISOLTRAP results and model predictions for the copper isotopes. Note that ${ }^{76} \mathrm{Cu}$ was measured for the first time and that the more recent parameter fit for HFB-8 included that result.

four previously unknown masses are also similar with DZ $(0.309 \mathrm{MeV})$, seeming to follow with a better trend than all the others (HFB-8: $0.400 \mathrm{MeV}$; HFB-2: $0.566 \mathrm{MeV}$; FRDM: $0.603 \mathrm{MeV}$ ). It is surprising that despite all models having their parameters adjusted to the mass tables that included those nuclides with $N<43$, those masses are not very well reproduced locally.

Some nucleon-nucleon effective interactions-for instance, Skyrme SKM*, SLy4, or Gogny D1-are designed to give rise to a realistic mean field (including pairing). They are therefore parametrized on the ground of a few available nuclear data for which mean field (including pairing) effects can be reasonably disentangled from long-range correlation effects (for instance, binding energies of doubly magic nuclei only). Such approaches of nuclei in which long-range correlations are not introduced in the mean field in an effective and somewhat uncontrolled manner do not have the objective of giving a precise mass formula at the mean (HFB) level (including pairing), but rather they aim to constitute the mean field input of more elaborate descriptions of nuclei by considering — at least some-long-range correlations up to the best, and therefore they are able to describe "beyond" the mean field a large class of nuclear observables (not only mass formula but also low-energy spectroscopy, shape coexistence, transitions, etc.).

In this frame, we have performed triaxial HFB calculations, using numerical methods and codes described in Ref. [64], with the Gogny-D1S force [65-67]. Figure 7 (left) presents the differences between the measured $\mathrm{Ni}$ masses and those predicted by HFB-D1S, as a function of $N$. There is a large offset (rms difference of $2.473 \mathrm{MeV}$ ) for the HFB-D1S masses, expected, as explained above, especially for midshell nuclei where long-range correlations play an important role. Under these assumptions, we could expect at least that the derivative

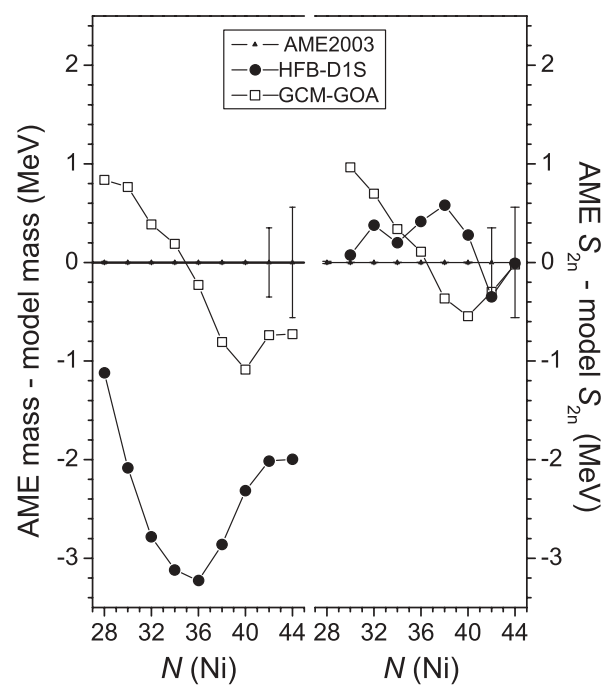

FIG. 7. Difference of the nickel results from the AME2003 data which already include the present ISOLTRAP data and those predicted by HFB-D1S (Gogny) and GCM-GOA as a function of neutron number $N$ for (left) the mass and (right) the two-neutron separation energy.

of these quantities might be closer to reality. Therefore, in Fig. 7 (right), we plotted the two-neutron separation energy $S_{2 n}$ [see Eq. (7)] derived from the same results. The result is encouraging, with an rms deviation of only $0.508 \mathrm{MeV}$.

In general, because of the existence of long-range correlations beyond the mean field, a unique HFB wave function is not well suited to describing the nuclear system. Thus, a configuration mixing approach already described and applied with some noticeable successes to different nuclear problems, for instance, to shape coexistence and transitions in light mercury isotopes [68] or to normal-super-deformed phenomena [69,70], has been considered. Using a generator coordinate approach under a Gaussian overlap approximation (GCM-GOA) in a space constituted by HFB (D1S) states under axial and triaxial quadrupole constraints allows us to treat rotation and quadrupole vibrations on the same footing in this model. This approach, which takes explicitly into account these important correlations, has been applied to the calculation of nickel masses, and the results are shown in Fig. 7 for comparison. Already the mass values (left) are greatly improved (rms difference of $0.701 \mathrm{MeV}$ ), as are the mass derivatives (right, rms difference of $0.335 \mathrm{MeV}$ ). It would appear that going beyond the mean field is to be encouraged for future mass predictions. Works in this spirit are also underway on the ground of Skyrme forces (see, e.g., Ref. [71]).

\section{ANALYSIS OF MASS SURFACE AROUND $Z=29$ AND $N=40$}

As recalled in the Introduction, Bohr and Mottelson [18] explain that the effects of binding energy on nuclear structure are subtle but decisive. As such, accurate mass measurements 


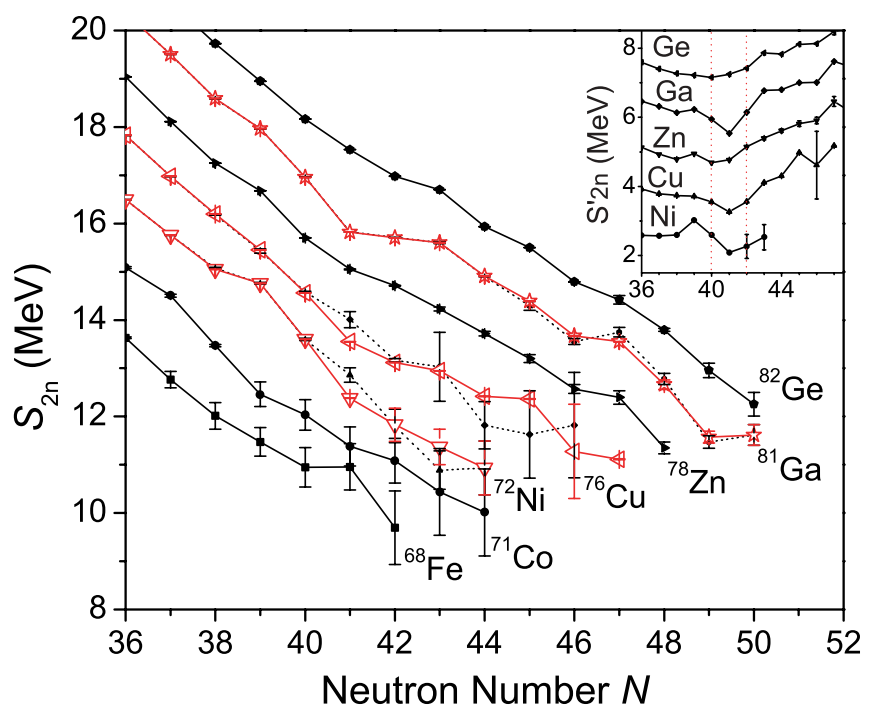

FIG. 8. (Color online) Two-neutron separation energies $\left(S_{2 n}\right)$ for iron $(Z=26)$ to germanium $(Z=32)$ around $N=40$. Dashed lines correspond to the data before the ISOLTRAP measurements. Points with large error bars were not directly measured by ISOLTRAP but their values were changed by the link to the measured masses. Inset represents $S_{2 n}^{\prime}$, which is $S_{2 n}$ minus a linear function of $N$, around $N=40$.

are important in order to finely analyze the mass surface, notably its derivatives. In this section, we examine several mass-surface derivatives and variations.

\section{A. Study of two-neutron separation energy}

The two-neutron separation energy $S_{2 n}$ given by

$$
S_{2 n}(N, Z)=B(N, Z)-B(N-2, Z),
$$

with $B$ for the binding energy, is remarkable for its regularity between shell closures. Generally, $S_{2 n}$ decreases smoothly with $N$, and shell effects appear as discontinuities. In the past, discontinuities of $S_{2 n}$ vs $N$ were often traced to inaccurate $Q_{\beta}$ endpoint measurements, and measurements with more reliable, direct techniques restored the regularity (see, for example, Ref. [72] for the area around ${ }^{208} \mathrm{~Pb}$ ). Hence, part of the motivation was to confirm any mass-surface irregularities in the $N=40$ region. Figure 8 presents the $S_{2 n}$ values, from $N=36$ to 50 , prior to and after the ISOLTRAP mass measurements. Most of the irregularities, e.g., at $N=$ 41 for gallium, are confirmed. Moreover, the plot reveals a deviation from the linear trend between $N=39$ and $N=41$ for nickel, copper, and gallium. Also, irregularities for gallium ( $N=46-49)$ and copper $(N=43-46)$ are visible.

To study the structure more closely, we subtract a linear function of $N$ determined by the $S_{2 n}$ slope preceding the purported shell closure. The resulting reduced $S_{2 n}^{\prime}$ values are presented in the inset of Fig. 8 in the region of $N=40$. For the $N=82$ shell closure, a change of slope between $N=82$ and $N=84$ is expected. From this, we can analyze the behavior in the $N=40$ region: there is a similar effect between $N=39$ and $N=41$ where the break can be seen at
$N=39$ and not at $N=40$, which is surprising for an odd number. The magnitude of this decrease is far smaller (between $500 \mathrm{keV}$ and $1 \mathrm{MeV}$ ) than the one for the major shell closure at $N=82$ (around $4 \mathrm{MeV}$ ). A similar structure is seen between $N=39$ and $N=41$ for nickel, copper, and gallium; but this is not an indication of shell closure. It is strange that the same structure is visible for both nickel (even $Z$ ) and gallium (odd $Z$ ), whereas germanium is smooth, and little is seen in the case of zinc. Further measurements to reduce the uncertainty on the neighboring cobalt isotopes will be needed.

\section{B. Shell gap}

The neutron shell gap, defined as

$$
\begin{aligned}
\Delta_{N}(N, Z) & =S_{2 n}(N, Z)-S_{2 n}(N+2, Z) \\
& =2 B(N, Z)-B(N-2, Z)-B(N+2, Z),
\end{aligned}
$$

is a good indicator of shell strength. The shell gap definition is usually only valid for spherical nuclides, i.e., around magic numbers. Here, we examine the case of $N=40$ and also investigate how midshell gaps compare in strength and comportment. Figure 9, calculated from AME2003 data [40], shows the shell gap as a function of the proton number $Z$ for for various $N$. This highlights the large shell gap values for magic neutron numbers with peaks at magic $Z$. It also shows that for $N=50$, there is a peak at $Z=39$ and not at $Z=40$, which is known to be semimagic. This behavior is probably due to the odd-even effect in the two-proton separation energy $S_{2 p}$. Not surprisingly, the midshell gap $(N=39,66)$ energies are quite small. From this point of view,

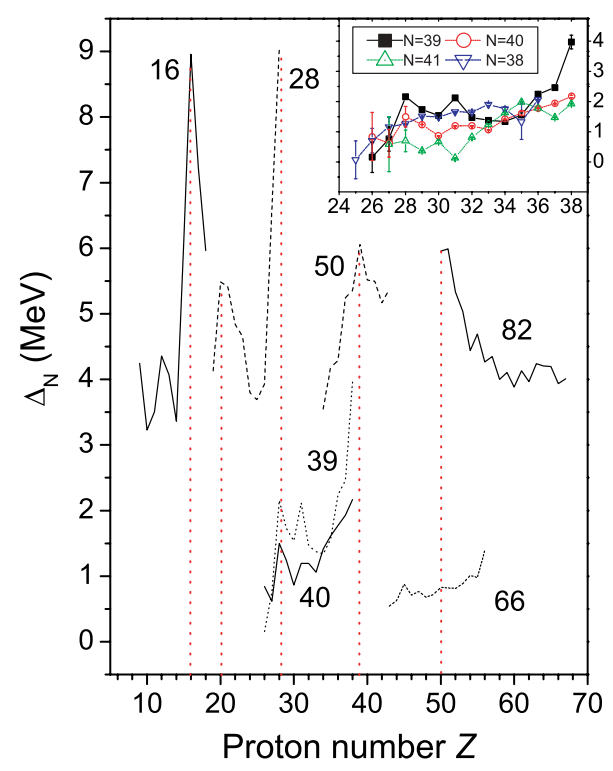

FIG. 9. (Color online) Shell gap as a function of proton number $Z$ for different magic and midshell neutron numbers. $N=$ $16,28,50,82$ correspond to shell closures; $N=39$ and 66 are exactly between two shell closures (called midshell), $N=40$ is under investigation. Data are from Ref. [40]. Inset represents a zoom for $N=38-41$. 
the case of $N=40$ resembles a midshell rather than a magic number.

The inset of Fig. 9 shows the details of adjacent shell gaps $\Delta_{N}$ as a function of the proton number $Z$ in the region of interest around $N=40$. For $N=40$, a strong difference (as expected for a shell closure) is not visible, and $N=40$ is distinct from neither $N=39$ nor 41 . Note that the $N=$ 39 midshell gap is larger than those of $N=38$ and 40 for several values of $Z$, especially for $Z=28$, unlike a midshell behavior. This shows that $N=38,39$, and 40 do not have the behavior we would have expected from observation in other mass regions. However, in summary, no shell closure at $N=40$ is observed.

\section{Pairing gap}

The pairing gap from the four-point formula [73]

$$
\begin{aligned}
\Delta_{4}(N, Z)= & \frac{(-1)^{N}}{4}[B(N+1) \\
& -3 B(N)+3 B(N-1)-B(N-2)]
\end{aligned}
$$

was chosen to study the pairing-energy behavior. A peak is expected for magic numbers and a trough at midshell.

The pairing gap as a function of neutron number is presented in Fig. 10(a) for $Z=28-32$. At the $N=39$ midshell, there is a trough for $Z=31$, but not for $Z=29$. A similar behavior is seen at $N=66$ (82-50 midshell). The odd- $Z$ nuclides have a lower pairing gap, and while germanium $(Z=32)$ shows no particular structure, nickel $(Z=28)$ shows a strong midshell trough and not a peak that would indicate a shell closure, as shown in Fig. 10(b) where shell closures at $N=28,50$, and 82 are clearly visible. The small energy scale associated with

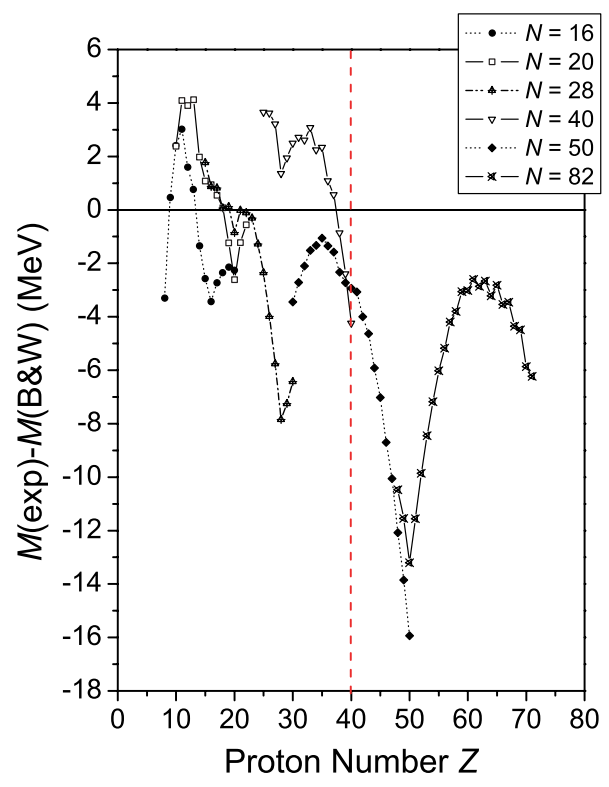

FIG. 11. (Color online) Difference between the experimental mass values from this work and from AME2003 data [40] and theoretical masses from the Bethe-Weizsäcker formula as a function of proton number, for several magic neutron numbers and for $N=40$.

the effects in Fig. 10(b) nicely illustrates the importance of mass measurements with very high precision.

\section{Comparison with Bethe-Weizsäcker formula}

The Bethe-Weizsäcker formula was given in Eq. (5). We adapt the version of Pearson [74], with a pairing term of Fletcher [75]. Thus, the binding energy per nucleon is given
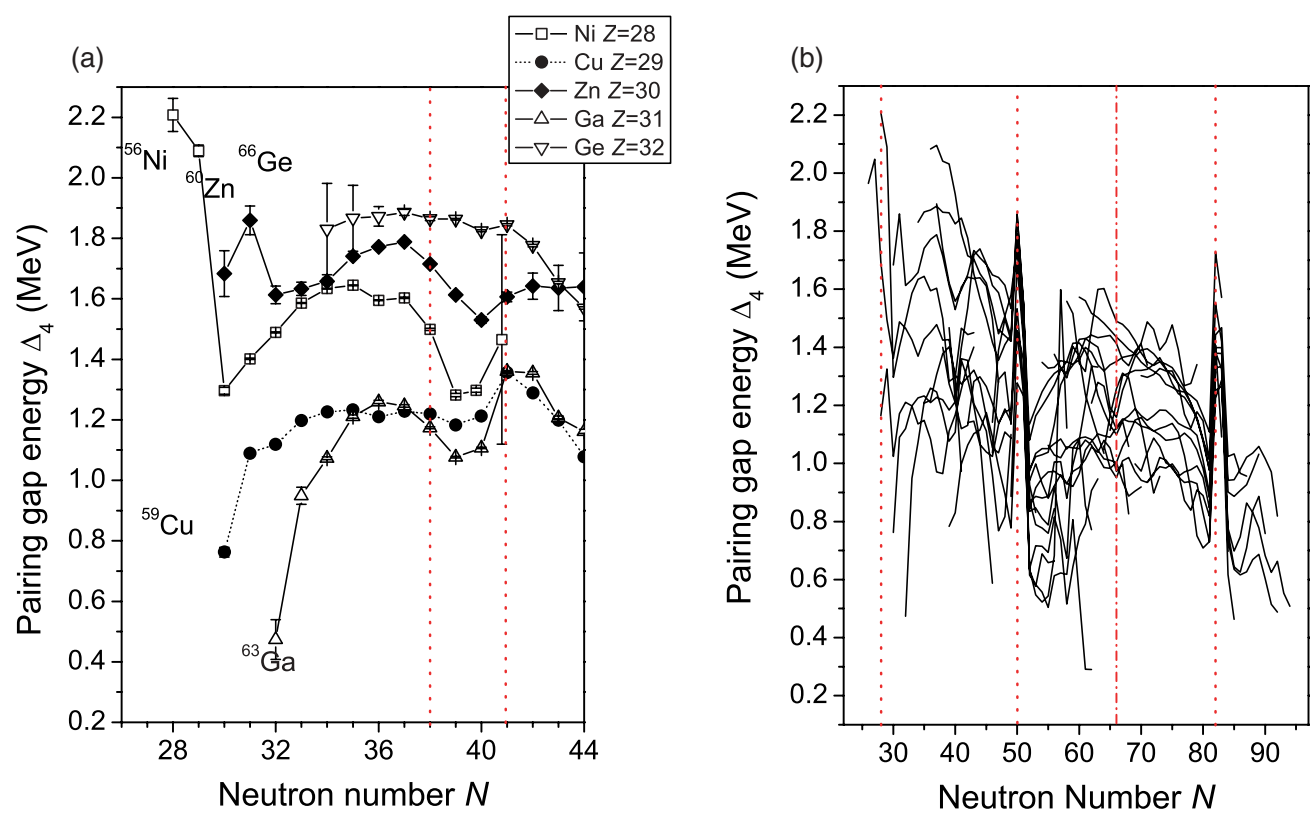

FIG. 10. (Color online) (a) Pairing gap energy as a function of neutron number for the investigated elements as well as for zinc and germanium. In the case of nickel, errors above $N=41$ are too large to be relevant, so the data are not shown. (b) Pairing gap energy as a function of neutron number for $Z=27-59$. Shell closures at $N=28,50$, and 82 are clearly visible; the $N=66$ midshell is indicated. 


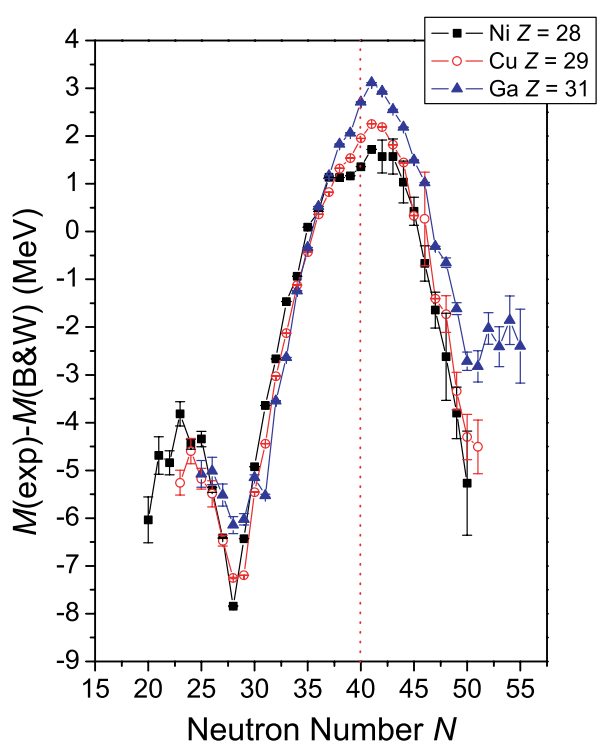

FIG. 12. (Color online) Difference between the masses predicted by the Bethe-Weizsäcker formula [Eq. (10)] and the experimental values as a function of $N$ for $Z=28,29$, and 31. Data are from this work, complemented by data from Ref. [40].

by

$$
\begin{aligned}
\frac{E_{\mathrm{nuc}}}{A}= & a_{\mathrm{vol}}+a_{\mathrm{sf}} A^{-1 / 3}+\frac{3 e^{2}}{5 r_{0}} Z^{2} A^{-4 / 3} \\
& +\left(a_{\mathrm{sym}}+a_{\mathrm{ss}} A^{-1 / 3}\right) I^{2} \\
& +a_{p} A^{-y-1}\left(\frac{(-1)^{Z}+(-1)^{N}}{2}\right),
\end{aligned}
$$

with $I=(N-Z) / A$. The parameters are $a_{\mathrm{vol}}=$ $-15.65 \mathrm{MeV}, a_{\mathrm{sf}}=17.63 \mathrm{MeV}, a_{\mathrm{ss}}=-25.60 \mathrm{MeV}$ (which is the parameter of the surface symmetry term introduced by Myers and Swiatecki [76]), $a_{\text {sym }}=27.72 \mathrm{MeV}, r_{0}=$ $1.233 \mathrm{fm}$ with $r_{0}$ the constant used in the radius estimation $R \approx r_{0} A^{1 / 3}, a_{p}=-7 \mathrm{MeV}$ the pairing term, and $y=0.4$. This formula contains no specific term for shell effects, so the formula may not be a good way to predict exotic mass values. However, this makes it a "neutral" indicator for shell structures (see Ref. [77]).

To this end, the modified Weizsäcker formula [Eq. (10)] is subtracted from known masses (divided by $A$ ). The difference between the experimental values and the formula clearly reveals the shell closures at $N=28,50,82$, and 126, reaching up to $15 \mathrm{MeV}$ for $N=50$ and $N=82$ (see Fig. 1 in Ref. [74]).

Figure 11 presents the difference between the experimental results obtained from this work (complemented with AME2003 data) and those calculated with the BetheWeizsäcker formula [Eq. (10)] as a function of $Z$ for various magic neutron numbers, including $N=40$. As with the shell gaps, the cases where $N=Z$ show the strongest effects, as does the case of ${ }_{50}^{132} \mathrm{Sn}_{82}$. Interestingly enough, the case of ${ }_{28}^{68} \mathrm{Ni}_{40}$ does show a dip of about $2 \mathrm{MeV}$, although it is only about $20 \%$ of the effect of ${ }_{50}^{132} \mathrm{Sn}_{82}$.

When the difference in mass values is examined isotopically as a function of neutron number (Fig. 12), however, there is no indication of a shell, or even subshell closure. The pseudoparabolic behavior of the curve in Fig. 12 shows some indentation around $N=40$ but nothing that we could claim to be "magic."

\section{CONCLUSION}

The high-precision mass measurements performed at ISOLTRAP on over 30 short-lived neutron-rich isotopes of nickel, copper, and gallium have allowed us to rather finely study the mass surface-and its derivatives-around the interesting region of $Z=28$ and $N=40$. No behavior resembling that of known magic numbers has been found, unlike the analog case of $Z=40$, where the $N=56$ subshell closure is visible. As much as an $N=40\left(d_{5 / 2}\right)$ subshell could exist, there is no clear indication for such a subshell closure from these measurements. While the pairing gap energy clearly indicates that there is no shell closure in this region, a competing midshell stabilization effect might be present. The comparison with the Bethe-Weizsäcker formula shows some fine structure around $N=39,40$ but no indication of the presence of a shell or subshell closure. The shell gap evaluation shows anomalous behavior for $N=39$ as well as for $N=40$, perhaps due again to the competition between a subshell closure at 40 and the midshell at 39 .

Recalling again the words of Bohr and Mottelson, "it is relatively difficult to discern the nuclear shell structure as long as the main information on nuclei is confined to binding energies." While they are a necessary ingredient, they are not sufficient for explaining the problem at hand, since the binding energies are in opposition with results on the $B(E 2)$ [16]. Thus, more detailed spectroscopy measurements, including the $g$ factor, as suggested by Langanke et al. [17], and more theoretical work are called for to understand the various phenomena arising from mass-surface studies.

\section{ACKNOWLEDGMENTS}

The authors thank the ISOLDE technical group for their assistance and acknowledge the GDR Noyaux Exotiques. This work was supported by the German Federal Ministry for Education and Research (BMBF) Contract No. 06GF151, Helmholtz Association of National Research Centers (HGF) Contract No. VH-NG-037, European Commission Contract No. HPRI-CT-2001-50034 (NIPNET), ISOLDE Collaboration, Marie Curie fellowship network HPMT-CT-2000-00197, and French IN2P3.
[1] C. Thibault, R. Klapisch, C. Rigaud, A. M. Poskanzer, R. Prieels, L. Lessard, and W. Reisdorf, Phys. Rev. C 12, 644 (1975).
[2] D. Guillemaud-Mueller, C. Detraz, M. Langevin, F. Naulin, M. De Saint-Simaon, C. Thibault, F. Touchard, and M. Epherre, Nucl. Phys. A426, 37 (1984). 
[3] A. Ozawa, T. Kobayashi, T. Suzuki, K. Yoshida, and I. Tanihata, Phys. Rev. Lett. 84, 5493 (2000).

[4] S. Shimoura, A. Saito, T. Minemura, Y. U. Matsuyama, H. Baba, H. Akiyoshi, N. Aoi, T. Gomi, Y. Higurashi, K. Ieki et al., Phys. Lett. B560, 31 (2003).

[5] F. Sarazin, H. Savajols, W. Mittig, F. Nowacki, N. A. Orr, Z. Ren, P. Roussel-Chomaz, G. Auger, D. Baiborodin, A. V. Belozyorov et al., Phys. Rev. Lett. 84, 5062 (2000).

[6] K. Minamisono, P. F. Mantica, T. J. Mertzimekis, A. D. Davies, M. Hass, J. Pereira, J. S. Pinter, W. F. Rogers, J. B. Stoker, B. E. Tomlin et al., Phys. Rev. Lett. 96, 102501 (2006).

[7] J. I. Prisciandaro, P. F. Mantica, B. A. Brown, D. W. Anthony, M. W. Cooper, A. Garcia, D. E. Groh, A. Komives, W. Kumarasiri, P. A. Lofy et al., Phys. Lett. B510, 17 (2001).

[8] P. F. Mantica, B. A. Brown, A. D. Davies, T. Glasmacher, D. E. Groh, M. Horoi, S. N. Liddick, D. J. Morrissey, A. C. Morton, W. F. Mueller et al., Phys. Rev. C 67, 014311 (2003).

[9] P. F. Mantica, A. C. Morton, B. A. Brown, A. D. Davies, T. Glasmacher, D. E. Groh, S. N. Liddick, D. J. Morrissey, W. F. Mueller, H. Schatz et al., Phys. Rev. C 68, 044311 (2003).

[10] M. Bernas, P. Dessagne, M. Langevin, J. Payet, F. Pougheon, and P. Roussel, Phys. Lett. B113, 279 (1982).

[11] L. P. Ekstrom and J. Lyttkens-Linden, Nucl. Data Sheets 67, 579 (1992).

[12] R. Broda, B. Fornal, W. Kròlas, T. Pawlat, D. Bazzacco, S. Lunardi, C. Rossi-Alvarez, R. Menegazzo, G. de Angelis, P. Bednarczyk et al., Phys. Rev. Lett. 74, 868 (1995).

[13] R. Grzywacz, R. Béraud, C. Borcea, A. Emsallem, M. Glogowski, H. Grawe, D. Guillemaud-Mueller, M. Hjorth-Jensen, M. Houry, M. Lewitowicz et al., Phys. Rev. Lett. 81, 766 (1998).

[14] M. Hannawald, T. Kautzsch, A. Wöhr, W. B. Walters, K.-L. Kratz, V. N. Fedoseyev, V. I. Mishin, W. Böhmer, B. Pfeiffer, V. Sebastian et al., Phys. Rev. Lett. 82, 1391 (1999).

[15] W. F. Mueller, B. Bruyneel, S. Franchoo, H. Grawe, M. Huyse, U. Köster, K.-L. Kratz, K. Kruglov, Y. Kudryavtsev, B. Pfeiffer et al., Phys. Rev. Lett. 83, 3613 (1999).

[16] O. Sorlin, S. Leenhardt, C. Donzaud, J. Duprat, F. Azaiez, F. Nowacki, H. Grawe, Z. Dombrádi, F. Amorini, A. Astier et al., Phys. Rev. Lett. 88, 092501 (2002).

[17] K. Langanke, J. Terasaki, F. Nowacki, D. J. Dean, and W. Nazarewicz, Phys. Rev. C 67, 044314 (2003).

[18] A. Bohr and B. R. Mottelson, Nuclear Structure (Benjamin, New York, 1969), Ch. 2, Sec. 2, p. 189.

[19] H. L. Seifert, J. M. Wouters, D. J. Vieira, H. Wollnik, X. G. Zhou, X. L. Tu, Z. Y. Zhou, and G. W. Butler, Z. Phys. A 349, 25 (1994).

[20] Y. Bai, D. J. Vieira, H. L. Seifert, and J. M. Wouters, AIP Conf. Proc. 455, 90 (1998).

[21] K. Blaum, Phys. Rep. 425, 1 (2006).

[22] L. Schweikhard and G. Bollen (ed.), Int. J. Mass Spectrom. 251(2/3) (2006).

[23] G. Bollen, P. Dabkievicz, P. Egelhof, T. Hilberath, H. Kalinowsky, F. Kern, H.-J. Kluge, R. B. Moore, H. Schnatz, L. Schweikhard et al., J. Appl. Phys. 68, 4355 (1990).

[24] H. Stolzenberg, S. Becker, G. Bollen, F. Kern, H.-J. Kluge, T. Otto, G. Savard, L. Schweikhard, G. Audi, and R. B. Moore, Phys. Rev. Lett. 65, 3104 (1990).

[25] E. Kugler, Hyperfine Interact. 129, 23 (2000).

[26] F. Herfurth, J. Dilling, A. Kellerbauer, G. Bollen, S. Henry,
H.-J. Kluge, E. Lamour, D. Lunney, R. B. Moore, C. Scheidenberger et al., Nucl. Instrum. Methods A 469, 254 (2001).

[27] H. Raimbault-Hartmann, D. Beck, G. Bollen, M. König, H.-J. Kluge, E. Schark, J. Stein, S. Schwarz, and J. Szerypo, Nucl. Instrum. Methods B 126, 378 (1997).

[28] G. Savard, S. Becker, G. Bollen, H.-J. Kluge, R. B. Moore, T. Otto, L. Schweikhard, H. Stolzenberg, and U. Wiess, Phys. Lett. A158, 247 (1991).

[29] L. S. Brown and G. Gabrielse, Rev. Mod. Phys. 58, 233 (1986).

[30] M. König, G. Bollen, H.-J. Kluge, T. Otto, and J. Szerypo, Int. J. Mass Spectrom. Ion Process. 142, 95 (1995).

[31] K. Blaum, G. Bollen, F. Herfurth, A. Kellerbauer, H.-J. Kluge, M. Kuckein, S. Heinz, P. Schmidt, and L. Schweikhard, J. Phys. B 36, 921 (2003).

[32] G. Bollen, H. Hartmann, H.-J. Kluge, M. König, T. Otto, G. Savard, and H. Stolzenberg, Phys. Scr. 46, 581 (1992).

[33] G. Bollen, S. Becker, H.-J. Kluge, M. König, R. B. Moore, T. Otto, H. Raimbault-Hartmann, G. Savard, L. Schweikhard, H. Stolzenberg et al., Nucl. Instrum. Methods A 368, 675 (1996).

[34] C. Yazidjian, K. Blaum, R. Ferrer, F. Herfurth, A. Herlert, and L. Schweikhard, Hyperfine Interact. (to be published).

[35] G. Gräff, H. Kalinowsky, and J. Traut, Z. Phys. A 297, 35 (1980).

[36] A. Kellerbauer, K. Blaum, G. Bollen, F. Herfurth, H.-J. Kluge, M. Kuckein, E. Sauvan, C. Scheidenberger, and L. Schweikhard, Eur. Phys. J. D 22, 53 (2003).

[37] U. Köster, Nucl. Phys. A701, 441c (2002).

[38] M. P. Bradley, J. V. Porto, S. Rainville, J. K. Thompson, and D. E. Pritchard, Phys. Rev. Lett. 83, 4510 (1999).

[39] A. H. Wapstra, G. Audi, and C. Thibault, Nucl. Phys. A729, 129 (2003).

[40] G. Audi, A. H. Wapstra, and C. Thibault, Nucl. Phys. A729, 337 (2003).

[41] G. Audi and A. H. Wapstra, Nucl. Phys. A595, 409 (1995).

[42] Ph. Dessagne, M. Bernas, M. Langevin, G. C. Morrison, J. Payet, F. Pougheon, and P. Roussel, Nucl. Phys. A426, 399 (1984).

[43] U. Köster, Ph.D. thesis, University of Munich, 2001.

[44] J. Van Roosbroeck, C. Guénaut, G. Audi, D. Beck, K. Blaum, G. Bollen, J. Cederkall, P. Delahaye, H. De Witte, D. Fedorov et al., Phys. Rev. Lett. 92, 112501 (2004).

[45] K. Blaum, D. Beck, G. Bollen, P. Delahaye, C. Guénaut, F. Herfurth, A. Kellerbauer, H.-J. Kluge, D. Lunney, S. Schwarz et al., Europhys. Lett. 67, 586 (2004).

[46] D. Lunney, J. M. Pearson, and C. Thibault, Rev. Mod. Phys. 75, 1021 (2003).

[47] C. F. von Weizsäcker, Z. Phys. 96, 431 (1935).

[48] H. A. Bethe and R. F. Bacher, Rev. Mod. Phys. 8, 82 (1936).

[49] P. Möller, J. R. Nix, W. D. Myers, and W. J. Swiatecki, At. Data Nucl. Data Tables 59, 185 (1995).

[50] J. Duflo and A. P. Zuker, Phys. Rev. C 59, R2347 (1999).

[51] E. Caurier, G. Martínez-Pinedo, F. Nowacki, A. Poves, J. Retamosa, and A. P. Zuker, Rev. Mod. Phys. 77, 427 (2005).

[52] H. Flocard, S. E. Koonin, and M. S. Weiss, Phys. Rev. C 17, 1682 (1978).

[53] P. Bonche, J. Dobaczewski, H. Flocard, P.-H. Heenen, and J. Meyer, Nucl. Phys. A510, 466 (1990).

[54] P.-H. Heenen, P. Bonche, and H. Flocard, Nucl. Phys. A588, 490 (1995).

[55] P. Bonche, H. Flocard, and P.-H. Heenen, Comput. Phys. Commun. 171, 49 (2005).

[56] F. Tondeur, S. Goriely, J. M. Pearson, and M. Onsi, Phys. Rev. C 62, 024308 (2000). 
[57] S. Goriely, F. Tondeur, and J. M. Pearson, At. Data Nucl. Data Tables 77, 311 (2001).

[58] M. Samyn, S. Goriely, P.-H. Heenen, J. M. Pearson, and F. Tondeur, Nucl. Phys. A700, 142 (2001).

[59] S. Goriely, M. Samyn, P.-H. Heenen, J. M. Pearson, and F. Tondeur, Phys. Rev. C 66, 024326 (2002).

[60] M. Samyn, S. Goriely, and J. M. Pearson, Nucl. Phys. A725, 69 (2003).

[61] S. Goriely, M. Samyn, M. Bender, and J. M. Pearson, Phys. Rev. C 68, 054325 (2003).

[62] M. Samyn, S. Goriely, M. Bender, and J. M. Pearson, Phys. Rev. C 70, 044309 (2004).

[63] S. Goriely, M. Samyn, J. M. Pearson, and M. Onsi, Nucl. Phys. A750, 425 (2005).

[64] M. Girod and B. Grammaticos, Phys. Rev. C 27, 2317 (1983).

[65] J. Dechargé and D. Gogny, Phys. Rev. C 21, 1568 (1980).

[66] J. F. Berger, M. Girod, and D. Gogny, Comput. Phys. Commun. 63, 107 (1991).

[67] D. Gogny, in Proceedings of the International Conference on Nuclear Physics, Munich, 1973, edited by J. De Boer and H. J. Mang (North Holland, Amsterdam, 1973); in Proceedings of the International Conference on Nuclear Selfconsistent fields,
Trieste, 1975, edited by G. Ripka and M. Porneuf (North Holland, Amsterdam, 1975).

[68] J. P Delaroche, M. Girod, G. Bastin, I. Deloncle, F. Hannachi, J. Libert, M. G. Porquet, C. Bourgeois, D. Hojman, P. Kilcher et al., Phys. Rev. C 50, 2332 (1994).

[69] J. Libert, M. Girod, and J.-P. Delaroche, Phys. Rev. C 60, 054301 (1999).

[70] J. P Delaroche, M. Girod, H. Goutte, and J. Libert, Nucl. Phys. A771, 103 (2006).

[71] M. Bender, G. F. Bertsch, and P.-H. Heenen, Phys. Rev. Lett. 94, 102503 (2005).

[72] S. Schwarz, F. Ames, G. Audi, D. Beck, G. Bollen, C. D. Coster, J. Dilling, O. Engels, R. Fossion, J.-E. G. Ramos et al., Nucl. Phys. A693, 533 (2001).

[73] J. Bardeen, L. N. Cooper, and J. R. Schrieffer, Phys. Rev. 108, 1175 (1957).

[74] J. M. Pearson, Hyperfine Interact. 132, 59 (2001).

[75] J. M. Fletcher, Masters thesis, University of Surrey (2003).

[76] W. D. Myers and W. J. Swiatecki, Nucl. Phys. 81, 1 (1966).

[77] C. Guénaut, G. Audi, D. Beck, K. Blaum, G. Bollen, P. Delahaye, F. Herfurth, A. Kellerbauer, H.-J. Kluge, D. Lunney et al., Eur. Phys. J. A Direct 25, s1.33 (2005). 